Frank Schulz-Nieswandt

\title{
Das Projekt Gemeindeschwesterplus in Rheinland-Pfalz im Kontext der kommunalen Daseinsvorsorge des bundesdeutschen sozialen Gewährleistungsstaatesplus
}

\section{Das Thema und die Struktur der Argumentation}

Zunächst wird in Abschnitt 1 nach einer Zusammenfassung der Ergebnisse der Evaluation ${ }^{1}$ (Abschnitt I) die Problematik lokaler Strukturen der Sozialraumbildung in rechtlicher Perspektive in angemessener Komplexität hergeleitet (Abschnitt II). Sodann (Abschnitt III) werden Überlegungen zur Finanzierbarkeit der Gemeindeschwester ${ }^{\text {plus }}$ dargelegt.

\section{Fragestellung}

Sollen Sozialversicherungen soziale Gebilde wie die der Gemeindeschwester ${ }^{\text {plus }}$ finanzieren? Es geht dabei um die grammatische, auf die Generik abstellende Logik solcher Gebilde und der Rolle der Sozialversicherungen im kommunalen Kontext der sozialen Daseinsvorsorge. ${ }^{2}$ Hier finanziert die Kasse nicht die individuelle, d. h. personenzentrierte Inanspruchnahme einer medizinischen Leistung gemäß Leistungskatalog, sondern investiert in lokale Strukturen der Generierung von Sozialkapital. Das drückt eine signifikant bedeutsame Differenz aus. Das ist hier sogar die Schlüsselfrage. Ist die Sozialversicherung als Versicherung für individuumszentrierte Einzelleistungskataloggewährung $\mathrm{zu}$ verstehen? Oder ist Sozialversicherung als para-fiskalische öffentlich-rechtliche Körperschaft im Rahmen der staatlichen Delegation öffentlicher Aufgaben im Sinn legitimer Instrumentalfunktion nicht auch verantwortlicher Mitspieler in der Daseinsvorsorge, die eben auch auf die Gewährleistung lokaler Strukturen der Generierung von Sozialraumentwicklung im Kontext regionaler Versorgungslandschaften abstellen muss? Ja, das ,?“ ist durch ein ,,!“ zu ersetzen.

1 Schulz-Nieswandt, F., Köstler, U. \& Mann, K. (2018), Evaluation des Modellprojekts „Gemeindeschwester ${ }^{\text {plus، }}$ des Landes Rheinland-Pfalz im Auftrag des Ministeriums für Soziales, Arbeit, Gesundheit und Demografie (MSAGD) Rheinland-Pfalz. Köln. (zugänglich unter https://msagd.rlp.de/de/unsere-themen/aeltere-menschen/ gemeindeschwesterplus/).

2 Schulz-Nieswandt, F. (2018 a), Lokale generische Strukturen der Sozialraumbildung. $§ 20 \mathrm{~h} \mathrm{SGB} \mathrm{V} \mathrm{und} \S 45 \mathrm{~d}$ SGB XI im Kontext kommunaler Daseinsvorsorge. Baden-Baden: Nomos. 


\section{Grundannahme}

$\mathrm{Zu}$ Recht wird vielfach in der einschlägigen Literatur betont, dass die bundesdeutsche Verfassung ,ein sich selbst verwaltendes Sozialversicherungssystem“ präferiert (vgl. auch dazu einschlägig BVerfGE 75, 108, 146; BVerfGE 79, 87, 101). Einerseits. Andererseits hat es nie ein reines Prinzip der Versichertenselbstverwaltung gegeben. Sozialversicherungen können sich in einem ordnungspolitisch wohlverstandenen Sinne durchaus der kommunalen Daseinsvorsorgeproblematik öffnen. Umgekehrt sind die Handlungsspielräume der Kommunen begrenzt. Nicht der Bund (Art. 84 Abs. 1 S. 7 GG), wohl aber gemäß Art. 83 GG die Länder (unter der Bedingung der Achtung des Konnexitätsprinzips im finanzverfassungsrechtlichen Sinne) können die Kommunen zu Aufgaben verpflichten, sodann aber auch redlich ermächtigen und befähigen. Sonst bleibt es eine Chimäre. Ansonsten sind die Möglichkeiten der Kommunen verfassungsrechtlich (Art. 74, Nr. 7, 1 GG, Art. 87 Abs. 2 GG) begrenzt. Hier kann Bezug genommen werden auf den Kompetenztitel „Öffentliche Fürsorge“ in Art. 74 Nr. 7 GG.

Ausgangspunkt der Überlegungen ist die Hypothese, dass es (Grundannahme 1) nicht schwer fällt, den rechtlichen Begründungszusammenhang zur Rechtfertigung von lokalen Strukturen wie die der Gemeindeschwester ${ }^{\text {plus }}$ (Abschnitt IV) herzuleiten (Abschnitt V.1). Schwieriger ist (Grundannahme 2) es, konkrete Paragraphen zur Heranziehung der Antwort auf die Finanzierungsweise zu identifizieren (Abschnitt V.2).

\section{Zusammenfassung der Evaluationsergebnisse}

Der Evaluationsbericht ist in seinem Aufbau im Kern an den auftragsseitig definierten Evaluationszielen orientiert. Die Evaluationsziele decken die relevanten Fragen ab, deren Beantwortung eine differenzierte Gesamteinschätzung des Projekts und auf dieser Grundlage notwendige Empfehlungen ermöglicht. Diese kurze (und daher auch zwingend selektive) Zusammenfassung fokussiert auf einige zentrale empirische Befunde und skizziert die Empfehlungsperspektive.

Im Evaluationsbericht finden sich natürlich auch eine Darstellung des methodischen (weitgehend qualitativen) Designs, die Erläuterung der Evaluationslogik in Bezug auf das Projekt und der Rolle der wissenschaftlichen Begleitung des Projekts von der Gründungsphase und entlang der Implementationsentwicklung bis Ende 2018 durch das DIP.

Der Zwischen- und Abschlussbericht des Deutschen Institutes für angewandte Pflegeforschung e. V. (DIP) der Gründungsphase sind im Internet auf der Homepage des DIP bzw. des Ministeriums für Arbeit, Soziales, Gesundheit und Demografie Rheinland-Pfalz (MSAGD) zu finden. Der Evaluationsbericht ist ebenso im Internet der Öffentlichkeit zur Verfügung gestellt und ordnet das Projekt zugleich (knapp und dicht) in den größeren sozial- und gesellschaftspolitischen Kontext des Diskurses und der Praxisentwicklung zu einer Sozialraum-orientierten Fortentwicklung der Alltags-zentrierten Verbesserung der lokalen/regionalen Versorgung der älteren Bevölkerung im Lichte der normativ-rechtlich vorgegebenen Werte der Selbstbestimmung, Selbstständigkeit und Teilhabe der Person im Gemeinwesen ein. 


\section{Die zentralen Evaluationsdimensionen sind:}

Zugang zum Angebot und Nutzung des Angebots, Zielgruppendefinition und Zielgruppenerreichbarkeit, Qualifikation und Aufgabenfelder, Kooperation mit anderen Anbietern vor Ort, Trägerschaft, Wirksamkeit, Erfolg und Nachhaltigkeit des Ansatzes der Gemeindeschwester ${ }^{\text {plus. }}$

\section{Allgemeine Einschätzung:}

Der Evaluationsbericht hat auf der Grundlage der Analyse aller Modellkommunen nur in fallübergreifender Weise die Analyseergebnisse dargelegt. Differenzierungen zwischen den Modellkommunen bleiben anonymisiert.

Die Ziele sind von den Modellkommunen weitestgehend in positiv zu beurteilender Weise erarbeitet worden.

Das bedeutet nicht, wenn dieser Jargon wegen seiner alltäglichen Verstehbarkeit genutzt werden darf, das nicht noch „Luft nach oben“ ist. Dabei muss die grundlegende Erfahrung aus solchen komplexen Einpflanzungen und Reifungsentwicklungen von Pilotprojekten in einer vorgegebenen, von Institutionen und Akteuren, von Interessen und Haltungen geprägten Landschaft berücksichtigt werden, dass es noch viel längere Zeit benötigt, bis ein neuer innovativer Baustein in die lokalen/regionalen Versorgungsarchitekturen nachhaltig akzeptiert und sodann wirksam eingefügt ist. Demnach wäre ein längerer Zeitkorridor zur Implementierung des Beratungs- und Vernetzungsangebotes durch die Gemeindeschwester ${ }^{\text {plus }}$ wünschenswert, denn die Erfahrung zeigt, dass es viele Jahre braucht, bis ein neues innovatives Angebot in die lokalen/ regionalen Versorgungsarchitekturen nachhaltig akzeptiert, vernetzt und damit wirksam eingefügt werden kann. Oftmals sind Pilotgebilde zunächst Fremdkörper.

Skalierend betrachtet sind die Zieldimensionen, die in der Evaluation abgebildet werden, auch unterschiedlich weit in den einzelnen Modellkommunen erarbeitet worden. Auch zeichnen sich auf der Basis der fallübergreifenden Gemeinsamkeiten differenzierte Schwerpunkte im Tätigkeitsprofil der einzelnen Modellkommunen ab, die sich aber kohärent aus den lokalen/regionalen Kontexten ergeben. Allerdings sind die Differenzierungen auch gut verständlich mit Blick auf die Unterschiede der Modellkommunen als städtische oder ländliche Räume.

\section{Wichtige zentrale Befunde:}

Die Gemeindeschwester ${ }^{\text {plus }}$ arbeitet weitgehend komplementär und somit leistungserweiternd im Verhältnis zu den Pflegestützpunkten. Unproduktive Redundanzen bestehen weitgehend nicht.

Das Kooperationsverhältnis zu den Pflegestützpunkten musste allerdings auch erst organisch wachsen. Einiges „Rauschen“ zu Beginn der Einpflanzung der Gemeindeschwester ${ }^{\text {plus war }}$ durchaus gegeben. Sicherlich spielten hier Unsicherheiten und professionspolitische sowie institutionenhabituelle Bedenken in der Situationsdefinition eine Rolle. Die Kooperationskultur konnte sich aber in fast allen Fällen im Zuge der sich konkretisierenden und der somit anschaulich werdenden Arbeit und des Leistungsprofils der Gemeindeschwesterplus und des kommunikativen Austausches zwischen den beiden Funktionsgebilden stark verbessern. 
Trägerschaftlich sind die Gebilde mit Blick auf die kooperative Leistungsprozesssteuerung durchaus kompliziert. Nicht alle Akzeptanzprobleme der Gemeindeschwester ${ }^{\text {plus }}$ aus Sicht von Akteuren im räumlichen Umfeld konnten ausgeräumt werden.

Die Datenerhebungen des DIP zur Akzeptanz in der lokalen Bevölkerung konnte dagegen ein sehr positives Erwartungsbild zeichnen. Allerdings musste im konkreten Versorgungsfall oftmals die Erwartung korrigiert werden, die Gemeindeschwester ${ }^{\text {plus }}$ sei zentral auf z. B. medizinkomplementäre Behandlung und Pflege ausgerichtet. Dagegen sei in positiver Hinsicht der Befund hervorgehoben, dass das Kohärenzerleben älterer Menschen im lokalen Raum durch die Präsenz der Gemeindeschwester ${ }^{\text {plus }}$ gesteigert werden konnte. D.h. das Vertrauen und das Sicherheitsempfinden älterer Menschen im sozialen Raum konnte gesteigert werden.

Die Leistungskomplementarität ergibt sich aus der Fokussierung der Arbeit der Gemeindeschwester $^{\text {plus }}$ auf die vor-pflegerische Situation. Damit ist die Gemeindeschwester ${ }^{\text {plus }}$, der Idee bzw. der organisatorischen „Blaupause“ nach, dem Aufgabenfeld der Pflegestützpunkte leistungsrechtlich im Sinne des SGB XI vorgelagert, wenngleich die Pflegestützpunkte in ihrem Tätigkeitsfeld auch in Themenkomplexe des SGB V hineinreichen. Im Alltag ist diese Trennung ,vor“ versus ,nach“ Eintritt von Pflegebedürftigkeit in der konkreten Lebenslage der betroffenen Personen nicht immer in aller Reinheitskultur gegeben; auch die Fokussierung auf die besonders vulnerable Hochaltrigkeit (Personen im Alter von mehr als 80 Jahren) ist nicht immer in Reinheitskultur aufgrund der konkreten Bedarfslagen der Haushalte durchzuhalten, zumal sich bei Fallausgrenzungen auch versorgungsethische Konflikte ergeben können. Dies galt auch für die regionalen Tätigkeitsgrenzen der Versorgungsräume der jeweiligen Modellkommunen.

Das Tätigkeitsprofil der Gemeindeschwester ${ }^{\text {plus }}$ ist, abstrakt definiert, eigentlich analog zu den Soll-Vorstellungen eines optimalen Leistungsprofils von Pflegestützpunkten: Gemeint sind Aufgaben der Beratung und Fallsteuerung im sozialräumlichen Kontext von lokalen Netzwerkbildungen. Dabei kristallisierten sich im Zuge der soeben angesprochenen wachsenden reziproken Akzeptanz im Projektverlauf zwei bzw. drei Deutungsmuster heraus:

I) In Pflegestützpunkten, wo sich in Bezug auf die Relation von Ressourcen des Personals einerseits und Bedarf des Gemeinwesens andererseits Grenzen des Machbaren abzeichnen, wird das Personal der Gemeindeschwester ${ }^{\text {plus }}$ als Erweiterung der Handlungsmöglichkeiten begrüßt. II) Als Teil des Deutungsmuster I wurde die Möglichkeit einer optimalen Arbeitsteilung zwischen den beiden Funktionsgebilden erkannt. Vor allem der fachpflegeprofessionell fundierte präventive Hausbesuch, aber auch eine Reihe von alltagsbegleitenden und Sozialraum-bezogenen Aktivitäten der Gemeindeschwester ${ }^{\text {plus }}$ wurden als sinnvolle und notwendige Ergänzungen eingeschätzt.

Hier kristallisierte sich auch ein Deutungsmuster III als Ableitung aus dem Muster II heraus, wonach trotz der abstrakten Ähnlichkeit im Leistungsprofil eine signifikante Komplementarität erkannt wurde. Ein typisches Beispiel für diese Aktivität der Gemeindeschwester ${ }^{\text {plus }}$ ist die Achtsamkeit im Aufgreifen von Einsamkeitsproblemen in hochaltrigen Single-Haushalten und eine entsprechende Hilfestellung. Typisch ist aber auch die Aktivierung hochaltriger Personen in der Nutzung des öffentlichen Raumes im Wohnumfeld. In solchen Projekten kann z. B. die Selbstblockade von Männern bei der Nutzung eines Rollators abgebaut werden. Auch die eröffnende Bahnung der Zugangschancen zu Möglichkeiten der Verlustbewältigung durch Trauerar- 
beit gehört (jenseits der Praxisprofile der Pflegestützpunkte) zum Aktivitätsspektrum der Gemeindeschwester ${ }^{\text {plus }}$ in der kümmernden Alltagshilfe im Zuge des präventiven Hausbesuchs.

Die Zielgruppen konnten demnach erreicht werden. Schwieriger erweist sich die Erreichbarkeit von Menschen mit spezifischen Migrationshintergründen. Die Aktivitäten des präventiven Hausbesuchs setzen hierbei zunächst die Türöffnungen im Rahmen Sozialraum-orientierter Netzwerkarbeit voraus. Aber auch dies wurde, wo erforderlich, in Modellkommunen erkannt und strategisch angegangen.

Mit dem Thema des Leistungsprofils der Gemeindeschwesterplus hängt die Kontroverse um die Zugangsqualifikation und um den Weiterbildungs- bzw. Schulungsbedarf ab. Die Schulungsarbeit des DIP erwies sich als sehr achtsam in der Bedarfsanalyse und sensibel in der Erhebung von Bedürfniserfordernissen. Die Schulungsarbeit des DIP erfreute sich in den Befragungen einer hohen wertschätzenden Akzeptanz.

Mit Blick auf die präventiven Hausbesuche erweist sich die fachpflegerische Qualifikation als eine unabdingbare Notwendigkeit, da hier der medizinische Wissensbestand (z. B. Krankheitsbilder) ebenso zentral ist wie die pflegediagnostischen Kompetenzen. Ansonsten stellen sich Weiterqualifikationsbedarfe mit Blick auf sektorübergreifende sozialrechtliche Kenntnisse ebenso wie Kompetenzentwicklung im Bereich der Fallsteuerung und der Netzwerkarbeit heraus.

Generell zeigt sich, dass bei Erhöhung des multi-professionellen Personalbestands in der Kooperation zwischen der Gemeindeschwester ${ }^{\text {plus }}$ und den Pflegestützpunkten ein Optimierungsbedarf mit Blick auf die Transparenz in der Arbeitsteilung der Kernkompetenzen besteht, aber eben auch in Richtung auf die wechselseitig achtsame Wertschätzung und Zusammenarbeitsbereitschaft und -kompetenz in den Überlappungsbereichen der Tätigkeitsprofile. Dies erweist sich vor allem als hoch bedeutsam bei der Steuerung von längeren Fallgeschichten mit vorpflegerischen und später pflegerischen Episoden. Hier zeigen sich Fallkonferenzkulturen in der Arbeitsplanung als unbedingt relevant.

Die lokalen/regionalen Arbeiten an der Kooperationslandschaft der verschiedenen Akteure stellen sich als ein von der Gemeindeschwester ${ }^{\text {plus }}$ aufgegriffenes Thema des Zielsystems der Modellkommunen heraus, entpuppen sich allerdings erwartungsgemäß als schwieriges Terrain, sodass Tugenden von Offenheit, Achtsamkeit, Mut, langem Atem bzw. langen Zeithorizonten, Frustrationstoleranz und Phantasie erforderlich sind. Vertrauensbildung ist hierbei die grundlegende Voraussetzung. Gelingende Kommunikation ist dabei der Schlüsselmechanismus.

Die Effizienz des Arbeitens ist eine Herausforderung eines längeren Lernprozesses. Dazu bedarf es neben einem individuellen Selbstmanagement (im Sinne einer achtsamen Selbstsorge von Nähe und Distanz) einer guten Koordination der Teams, also einer guten Führung des Funktionsgebildes. Dies sind Erfordernisse der betrieblichen Prozesssteuerung, gerade auch unter Aspekten der Ziel-orientierten Kosten-Effektivität.

\section{Ausblick}

Die Erfolge, gerade auch die zielgruppenorientierte, bedarfsdeckende Wirksamkeit und ihre Nachhaltigkeit, der Gemeindeschwester ${ }^{\text {plus }}$ sind mit Blick auf die weitere Zukunft als vielver- 
sprechend einzuschätzen. Dennoch bleibt das Funktionsgebilde ein ständiger Organisationslernprozess, intern mit Blick auf die Personalaufstellung und die Leistungsprozesse, extern mit Blick auf die Veränderungen in der sozialräumlichen Umwelt dieser Aufgabenprofile. Auch die Aufgabenprofile und der entsprechende Qualifizierungsbedarf selbst sind Dimensionen des Lernens in einem nie vollständig abgeschlossenen Prozess.

\section{Verfassungsrechtliche Herleitung der Rolle der Länder und der Kommunen in der Pflegepolitik}

\section{a) Sozialer Bundesstaat als Schlüsselverankerung}

Die Bundesrepublik ist, nochmals ergänzend fundiert und konstitutionell gerahmt im Rahmen des Unionsbürger*innenrechts des EUV, gemäß GG als Rechtsstaat geordnet. Im Art. 20 GG wird diese Rechtsstaatlichkeit als sozialer Bundesstaat konkretisiert. Damit wird der bundesdeutsche Rechtsstaat als sozialer und föderaler Rechtsstaat konkretisiert.

Das Soziale in der adjektiven Bestimmung verweist auf den Sozialstaatscharakter als Materialisierung des Rechtsstaates. Subjektcharakter hat nur der Rechtsstaat (mit seinem Monopol auf legitime Gewalt); der Sozialstaat ist seine Ausdrucksform.

Die föderale Ordnung weist dem Bund und den Ländern Eigengesetzlichkeit zu. Die Kommune der kreisfreien Städte und der Landkreise sind ohne Eigengesetzlichkeit in diesem Rahmen zur kommunalen Daseinsvorsorge gemäß Art. 28 GG zu ermächtigen.

Ort des Geschehens der sozialen Daseinsvorsorge ist daher in der Regel der lokale bzw. regionale Lebenszusammenhang der Menschen im Alltag. Dies ist kongenial zur bundesdeutschen Raumordnungspolitik gemäß des Leitbildes ausgeglichener und integrierter Funktionsräume (abgestellt auf Arbeit und Wohnen, Mobilität und Erholung). Das Axiom der Gleichwertigkeit der Lebensverhältnisse im Raum in Art. 72 GG ist in diesem Sinne in Bezug auf die Aufgabe kommunaler Daseinsvorsorge zu lesen. Vor diesem Hintergrund ist das in Art. 36 der Grundrechtscharta der EU, primärrechtlich verankert in EUV und AEUV, als Grundrecht auf freien Zugang zu den Dienstleistungen von allgemeinem Interesse kodifiziert zu verstehen.

Die föderale Ordnung ist eine unitarische Ordnung, die von einem „echten“ Föderalismus abweicht, da Politikfelder und Rechtsmaterien nicht eindeutig auf Bundes- und Landesebene aufgeteilt sind, sondern es zu vertikalen Politikverflechtungen - nicht im engeren Sinne von Gemeinschaftsaufgaben - kommt. Bei Landeskompetenzen im jeweiligen Politikfeld wird insbesondere die Gesetzgebungslogik des Zwei-Kammer-Systems in Deutschland deutlich.

\section{b) Fundamentale Normwerte, die aus der Personalität des Menschen resultieren}

Vor dem Hintergrund des auch völkerrechtlich fundierten Axioms der Würde im personalen Menschenbild der Verfassung in Art 1. GG charakterisiert der Art. 2 GG mit dem Grundrecht auf eine freie Entfaltung der Persönlichkeit im gesamten Lebenslauf die freiheitliche Ordnung der Verfassung. 
Der $§ 1$ SGB I konkretisiert dies kohärent im Lichte sozialer Gerechtigkeit zugleich im gesamten Sozialrecht. Es ist unzweifelhaft evident, dass es um den fundamentalen Normwert der teilhabenden Selbstbestimmung im Modus der selbstverantwortlichen und sozial mitverantwortlichen (vgl. u. a. § 1 SGB V und § 6 SGB XI) Selbstständigkeit der Person geht. Dieser fundamentale Normwert als Ordnungsfluchtpunkt des GG und des gesamten Sozialrechts des Systems der SGB ist zugleich vom Prinzip der Subsidiarität als ,,(kollektive) Hilfe zur (individuellen) Selbsthilfe“ geprägt. So etwa mit Blick auf die Definition der Sozialhilfe im § 9 SGB I.

Gerade die Pflegepolitik - analog auch die Krankenhauspolitik - ist von der Bundesgesetzgebung einerseits und von der Politik der Bundesländer geprägt. So weist das SGB XI gemäß § 9 den Bundesländern die gesetzgeberische Konkretisierungsaufgabe in Bezug auf die Infrastruktur der pflegerischen Versorgungslandschaft zu. Die Länder greifen dies im Rahmen von Landespflegegesetzen sowie im entsprechenden Verordnungswesen auf, seit der Föderalismusreform 2006 auch im Rahmen der WTG, vor allem im Lichte von $§ 3$ SGB XI.

\section{c) Gewährleistung und Sicherstellung im Rahmen der Subsidiarität}

In diesem Rahmen der bundesdeutschen Ordnung fallen Gewährleistung und Sicherstellung der Daseinsvorsorge und des Sozialschutzes in der Regel auseinander.

Dies gilt einerseits mit Blick auf die Mehr-Ebenen-Schichtung von Bund, Länder und Kommunen. Dies gilt andererseits auch für die Nachrangigkeit öffentlicher Leistungsanbieter gegenüber der in (übergreifend europarechtlich) regulierten Quasi-Märkten wettbewerblich geordneten privaten (privatwirtschaftlichen) und freien (freigemeinwirtschaftlichen) Trägern der Leistungserstellung.

\section{d) Rolle der Kommunen}

Die Kommunen sind gemäß Art. 28 GG Ort des Daseinsvorsorgegeschehens und in diesem Zusammenhang als Träger ausgewiesen. Im Kontext von § 9 SGB XI vor dem Hintergrund der zentralen Hilfe-Mix-Idee des $\S 8$ (Pflege als gesamtgesellschaftliche Aufgabe) müssen Kommunen befähigt werden, im Sinne der Idee lokaler sorgender Gemeinschaften im Rahmen einer regionalen Pflegestrukturplanung (im Schnittbereich zu SGB V und SGB XI sowie SGB XII) eine nachhaltige Sozialraum-orientierte Lebenswelt der Menschen in ihren örtlichen Alltagsprozessen zu ermöglichen. Die Kommune ist hier als Sozialhilfeträger nicht nur mit Blick auf die Hilfe zur Pflege und der Eingliederungshilfe gefragt, sondern auch auf der Grundlage von $\S 71$ SGB XII in der Hilfe zum erfolgreichen Hineinaltern.

\section{e) Rolle der Sozialversicherungen}

Der Kern der Sozialversicherungen im Geltungsbereich von SGB V und SGB XI ist die Gewährleistung der Sicherstellung von Personen-zentrierten Einzelleistungen im Sinne des versi- 
cherungsrechtlichen Leistungskatalogs. Dazu gehören aber auch Aufgaben im Bereich der Beratung und der Fallsteuerung, die wiederum nicht ohne Förderung lokaler bzw. regionaler Netzwerkbildungen möglich sind.

Derartige Förderungen (Finanzierungen) lokaler Strukturen der Generierung der Sozialraumentwicklung sind dem Sozialrecht überhaupt nicht fremd. Hier sind einerseits die Pflegestützpunkte gemäß $\S 7$ c SGB XI anzuführen, aber auch die Finanzierung von Kontaktstellen der Selbsthilfeförderung im Sinne des $\S 20$ h SGB V sowie im Sinne des $§ 45$ d SGB XI (in Kooperation mit den Ländern).

\section{Perspektiven für die Finanzierung der Gemeindeschwester ${ }^{\text {plus }}$}

Die Sozialraumentwicklungsfinanzierung gemäß $\S 20$ h SGB V sowie $§ 7$ c sowie 45 d SGB XI machen deutlich, dass grundsätzlich eine Finanzierung der komplentären bzw. äqui-funktionalen Leistungen der Gemeindeschwester ${ }^{\text {plus }}$ aus Sicht der Sozialversicherung möglich wird. Hier ginge es einerseits um pflege- und gesundheitspräventive Funktionen, andererseits um begleitende Alltagshilfen, wie sie das neuere Sozialrecht an vielen Stellen kennt.

Da signifikante Schnittflächen mit kommunalen Pflicht- und Soll-Aufgaben (Soll-Aufgaben wie der $\S 71$ SGB XII sind keine freiwilligen Aufgaben) gegeben sind, ist eine Mischfinanzierung zwingend und im Grundsatz keineswegs verfassungswidrig, muss aber anreizkonform ausgestaltet werden, um unerwünschte strategisch-opportunistisches Verhalten zu vermeiden.

Auch die trägerschaftliche Aufhängung ist offen gestaltbar. Aus Gründen des Neutralitätsgebots wäre eine öffentlich/öffentlich-rechtliche Partnerschaft zwischen Kommunen und Sozialversicherung die an sich gebotene Lösung, mag aber aus Gründen der politischen Pfadabhängigkeiten nicht unbedingt realisierbar sein. Die reinste Form in diesem Sinne wäre eine gewichtete tripartistische Mischfinanzierung durch Kommune, Kranken- und Pflegeversicherung, aber mit kommunaler Aufhängung mit Blick auf die betriebliche Prozesssteuerung der Einrichtung und ihres Leistungsprofils im Sozialraum.

Ein solches Regie- und Finanzierungsmodell benötigt eine politische Einigung und somit Zeit. Insofern wäre der im Koalitionsvertrag ${ }^{3}$ angedeutete Vorschlag sinnvoll, auf der Basis des Bundespräventionsgesetzes (nunmehr geregelt in $\S \S 20 \mathrm{dff}$ SGB V) die Strukturentwicklung des präventiven Hausbesuchs im Zusammenhang mit alltagsbegleitenden Aktivitäten im Rahmen der lokalen Netzwerkarbeit unter der Bedingung der Optimierung der Schnittstellen zu Einrichtungen gemäß $\S 7 \mathrm{c}$ SGB XI sowie $\S 20 \mathrm{~h}$ SGB V und $\S 45 \mathrm{~d}$ SGB XI voranzutreiben. Die Schnittfläche der Hilfen bei Krankheit (SGB V), Pflege (SGB V) und Behinderung (SGB IX) sind zu gestalten.

In nachhaltiger Sicht müssen die Länder eigene kommunale Strukturen in diesem perspektivischen Sinne entwickeln.

3 Vgl. Schulz-Nieswandt, F. (2018b): Stellungnahme zur Alternspolitik im Koalitionsvertrag vom 7. Februar 2018. In: ProAlter, 50(2), S. 45-47. 


\section{Empfehlungen}

Solche lokalen Strukturen der Sozialraumbildung und der dort eingelassenen, auf das Gelingen der personalen Daseinsbewältigung abstellenden, orientierenden Beratungs- und Alltagsbegleitungsleistungen wie die der Gemeindeschwester ${ }^{\text {plus }}$, angesiedelt zwischen den Kernaufgaben der Krankenversicherung einerseits und der Pflegeversicherung anderseits, erweisen sich mit der Zunahme der vulnerablen Hochaltrigkeit in der Bevölkerung als notwendig, sinnvoll, zweckmäßig und erfolgsversprechend.

Die koordinierende Optimierung von Komplementärfunktionen zu anderen analogen Funktionsgebilden wie die Pflegestützpunkte nach $§ 7$ c SGV XI (aber auch mit Blick auf die Kontaktund Informationsstellen der Selbsthilfeförderung gemäß $§ 20$ h SGB V und $\S 45$ d SGB XI) erweist sich jedoch als außerordentlich wichtig im Lichte der Kosten-Effektivität. Dies ergibt sich aus dem gewollten Befund, dass die Gemeindeschwestern ${ }^{\text {plus }}$ in den Modellkommunen in Rheinland-Pfalz über den wichtigen Kern des fachpflegerisch fundierten präventiven Hausbesuchs hinausreichend wirken. Es ergeben sich Schnittflächen mit Aufgaben einer Alltagsbegleitung und mit Stadtteil- bzw. Dorfkümmererfunktionen.

Auch sind dabei Schnittflächen mit kommunalen Aufgaben (z. B. die Sollaufgabe der Altenhilfe gemäß § 71 SGB XII) gegeben. Schnittflächen ergeben sich auch mit der innovativen Reform der Präventionsaufgaben im SGB V durch das Lebenswelt-bezogene Paradigma des Bundespräventionsgesetzes.

Das Land, federführend durch das MSAGD, kommt durch die Finanzierung der Gemeindeschwestern $^{\text {plus }}$ in den Modellkommunen seinem Auftrag gemäß $§ 9$ SGB XI (Rolle der Länder bei der Gewährleistung moderner Versorgungslandschaften) nach und steht dabei auch in Übereinstimmung mit modernen fachpolitischen Einsichten wie z. B. mit den Analysen und Empfehlungen des 7. Altenberichts der Bundesregierung. Dauerhaft handelt es sich aber um Aufgaben auf der lokalen/regionalen Ebene im Rahmen der Sozial-raumorientierten kommunalen Daseinsvorsorge. Hier wird zu klären sein, wie sich eine Mischfinanzierung zwischen Kommunen und Sozialversicherungen herstellen lässt. Dabei müssen Kosten-effektive Steuerungsmodelle mit Blick auf die trägerschaftliche Aufhängung - insbesondere in Bezug auf die notwendigen Entscheidungen hinsichtlich der Kooperations- oder Integrationsmodelle mit den Pflegestützpunkten - ermöglicht werden. Die Frage, die auch in den Evaluationsbefunden offen und kontrovers geblieben ist, bleibt die nach der möglichen federführenden Rolle der Kommunen.

\section{Literaturverzeichnis}

Schulz-Nieswandt, F. (2018 a), Lokale generische Strukturen der Sozialraumbildung. $§ 20 \mathrm{~h}$ SGB V und $\S 45 \mathrm{~d}$ SGB XI im Kontext kommunaler Daseinsvorsorge. Baden-Baden: Nomos.

Schulz-Nieswandt, F. (2018 b), Stellungnahme zur Alternspolitik im Koalitionsvertrag vom 7. Februar 2018. In: ProAlter 50(2), S. 45-47.

Schulz-Nieswandt, F., Köstler, U. \& Mann, K. (2018), Evaluation des Modellprojekts „Gemeindeschwester ${ }^{\text {plus“ }}$ des Landes Rheinland-Pfalz im Auftrag des Ministeriums für Soziales, Arbeit, Gesundheit und Demografie (MSAGD) Rheinland-Pfalz. Köln. (zugänglich unter https://msagd.rlp.de/de/unsere-themen/aeltere-menschen/ gemeindeschwesterplus/). 